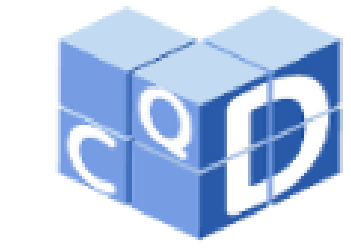

Revista Eletrônica

Paulista de Matemática

ISSN 2316-9664

Volume 15, jul. 2019

Jorge Corrêa de Araújo

FFP-UERJ

jcaraujo_55@yahoo.com.br

Rosa García Márquez

FFP-UERJ

rosagmarquez@yahoo.com.br

\section{Soluções de alguns problemas da equação de Burgers unidimensional}

\author{
Solutions of some problems of the one-dimensional \\ Burgers equation
}

\begin{abstract}
Resumo
Nesse artigo, é utilizada a transformação de Hopf-Cole para obter a solução da equação de Burgers unidimensional em alguns casos particulares de condição inicial, baseados na equação de condução de calor, nos domínios infinito, semiinfinito e finito até então não referenciados na literatura consultada sobre o assunto. Um exemplo particular da equação de Burgers com condições iniciais por partes foi resolvido como uma onda viajante usando a condição de Rankine-Hugoniot e também utilizando a transformação de Hopf-Cole, e deve enunciar de forma clara e sintética o problema de pesquisa, a abordagem metodológica empreendida, resultados e conclusões.
\end{abstract}

Palavras-chave: Equação de Burgers. Transformação de Cole. Domínio finito e infinito. Solução explícita. Onda viajante.

\begin{abstract}
In this paper, the Hopf-Cole transformation is used to obtain the solution of the one-dimensional Burgers equation in some particular cases of initial condition based on the heat conduction equation, in the infinite, semi-infinite and finite domains not previously referenced in the estimated literature about the subject. A particular example of the Burgers equation with initial conditions in parts was solved as a traveling wave using the Rankine-Hugoniot condition and also using the Hopf-Cole transformation.
\end{abstract}

Keywords: Burgers equation. Hopf-Cole transformation. Infinite e finite domain. Explicit solution. Travel wave. 


\section{Introdução}

A equação diferencial parcial (EDP) quase linear, de segunda ordem, parabólica unidimensional

$$
\left(\frac{\partial u}{\partial t}\right)+u\left(\frac{\partial u}{\partial x}\right)=v\left(\frac{\partial^{2} u}{\partial x^{2}}\right), \quad a<x<b, \quad t>0,
$$

onde $u=u(x, t)$ e $v$ é um parâmetro, é conhecida como equação de Burgers (KUTLUAY; BAHADIR; ÖZDEŞ, 1999, p. 252), sendo ela um caso particular do sistema de equações idealizado por Burgers (1948, p. 172-173) para formular um modelo matemático unidimensional de turbulência para ser usado em problemas de hidrodinâmica. Nessa equação, o parâmetro $v=\frac{1}{R}, R>0$ é o número de Reynolds que é usado para representar os efeitos friccionais devido ao movimento do fluido, seu valor indica se o fluxo é turbulento ou laminar. Segundo Dhawan, Kapoor, Kumar e Rawat (2012, p. 405) a equação de Burgers envolve simultaneamente os efeitos de propagação não linear e os efeitos difusivos representados respectivamente pelos termos $u\left(\frac{\partial u}{\partial x}\right)$ e $v\left(\frac{\partial^{2} u}{\partial x^{2}}\right)$. A equação de Burgers é similar às equações de Navier-Stokes, as quais segundo Fox, Mcdonald e Pritchard (2015, p. 199-200), constituem um conjunto de equações que são largamente utilizadas para descreverem escoamento de fluido com ênfase no movimento das partículas, o que torna a equação de Burgers ainda mais atraente. Para verificar a similaridade dessas equações basta considerar o escoamento como sendo unidimensional, incompressível, de viscosidade constante e gradiente de pressão nulo. $\mathrm{O}$ modelo de Burgers tem sido utilizado, segundo Dhawan, Kapoor, Kumar e Rawat (2012, p. 406), na propagação de ondas não lineares, ondas de choque, dinâmica de gases e choques de fluxos entre outras aplicações de importância.

$\mathrm{Na}$ equação (1) os termos $u, x, t$ e $v$ são respectivamente a velocidade do escoamento de fluido, a coordenada espacial, o tempo e o coeficiente de viscosidade cinemática. A equação de Burgers foi resolvida primeiramente por Hopf (1950, p. 204) no semiplano $t>0$ e depois por Cole (1951, p. 231), que apresentou um modelo teórico de decaimento de turbulência livre em uma caixa onde a condição inicial e de fronteira são dadas por

$$
u(x, 0)=u_{0}(x), \quad 0 \leq x \leq L
$$

$\mathrm{e}$

$$
u(0, t)=u(L, t)=0, \quad t>0 .
$$

A equação (1) pode ser resolvida mediante a transformação Hopf-Cole (KUTLUAY; BAHADIR; ÖZDEŞ, 1999, p. 253) dada por

$$
u=-\frac{2 v}{\theta}\left(\frac{\partial \theta}{\partial x}\right)
$$

onde $\theta=\theta(x, t)$ é a solução da EDP linear que governa problemas de condução de calor unidimensional dada por 


$$
\frac{\partial \theta}{\partial t}=v \frac{\partial^{2} \theta}{\partial x^{2}}
$$

sendo $u$ dado na equação (4) a solução da equação quase linear (1). Reciprocamente, se $u$ é solução da equação (1) então $\theta$ obtido da equação (4) é solução da equação de calor (5), a menos de um termo arbitrário multiplicativo dependente do tempo e que é irrelevante na equação (4) (BENTON; PLATZMAN, 1972, p. 195). A equação (1) depende essencialmente da condição inicial $u_{0}(x)$ onde figura na expressão da solução $u(x, t)$. A equação de Burgers é, segundo Bahadir, Kutluay e Özdeş (1999, p. 252), uma das poucas EDP’s não lineares que pode ser resolvida de forma exata, usando somente a condição inicial $u_{0}(x)$. Benton e Platzman (1972, p. 202-208) disponibilizaram uma tabela contendo trinta e cinco soluções explícitas distintas para as equações (1) e (5) com a condição inicial (2) em um domínio infinito.

Nesse trabalho revisamos de forma mais detalhada, a solução do primeiro exemplo dado por Cole (1951, p. 231-232) que trata de uma onda de choque que se aproxima rapidamente de um estado estacionário e que servirá de apoio para os exemplos aqui apresentados. Com base nessa revisão a solução da equação de Burgers com as condições (2) e (3) utilizando como condição inicial a função cosseno foi resolvida. Considerando apenas o primeiro termo da condição de fronteira $u(0, t)=0$ e a condição inicial $u_{0}(x)$, uma solução explícita da equação de Burgers em meio semi-infinito foi disponibilizada sendo que esse exemplo não consta da literatura disponível dos diversos autores citados nesse artigo. Uma equação de Burgers, com condição inicial constante por partes, foi resolvida detalhadamente usando a condição de Rankine-Hugoniot (CUMINATO; MENEGUETTE JUNIOR, 2013, p. 254-256) e a transformação de Hopf-Cole (KUTLUAY; BAHADIR; ÖZDEŞ, 1999, p. 253).

\section{Métodos}

A lei da conservação de massa, caso homogêneo, é expressa matematicamente na forma integral como (FOX; PRITCHARD; MCDONALD, 2015, p. 106)

$$
\frac{\partial}{\partial t} \int_{\Omega} \rho d \forall+\int_{\partial \Omega} \rho \vec{V} \vec{d} \sigma=0,
$$

sendo $\vec{d} \sigma=\vec{n} \cdot d \sigma$, onde $\vec{n}_{\mathrm{O}}$ vetor normal ao elemento de área $d \sigma$ da superfície $\partial \Omega$ da região $\Omega$ fixada do espaço tridimensional onde o fluído escoa, sendo $\Omega$ denominado volume de controle (VC). O primeiro termo integral da equação (6) é a taxa de variação de massa $\frac{d M}{d t}$ de uma substância de concentração $\rho=\rho(\vec{x}, t)$ dissolvida no fluído com campo de velocidade $\vec{V}=\vec{V}(\vec{x}, t)$. O produto $\rho \vec{V}$ é a densidade de fluxo de massa, isto é, é a quantidade de substância que atravessa uma unidade de área da superfície de controle por unidade de tempo. Se $\frac{\partial \rho}{\partial t}$ é contínua em $\Omega$, tem-se segundo Crowell, Trotter e Willianson (1972, p. 488) que 


$$
\frac{d M}{d t}=\int_{\Omega} \frac{\partial \rho}{\partial t} d \forall
$$

Das equações (6) e (7) e do teorema da divergência (GUIDORIZZI, 2019, p. 234) resulta a forma diferencial da equação de conservação de massa dada por

$$
\frac{\partial \rho}{\partial t}+\nabla \cdot \rho \vec{V}=0
$$

No caso unidimensional, a equação (8) fica na forma escalar dada pela equação diferencial parcial hiperbólica como

$$
\frac{\partial \rho}{\partial t}+\frac{\partial(\rho V)}{\partial x}=0
$$

Se $F(\vec{x}, t)$ é a taxa de criação ou destruição de massa, a lei de conservação de massa fica na forma

$$
\frac{\partial \rho}{\partial t}+\frac{\partial(\rho \vec{V})}{\partial x}=F(\vec{x}, t) .
$$

No caso unidimensional a equação (10) é dada por

$$
\frac{\partial \rho}{\partial t}+\frac{\partial(\rho V)}{\partial x}=F(x, t)
$$

No caso escalar, o termo $\rho V$ representa simplesmente o fluxo de massa, por exemplo, em $\frac{k g}{s}$. Em particular, se $\vec{V}=v \vec{i}$, onde $v$ é constante, a equação (9) pode ser escrita como

$$
\frac{\partial \rho}{\partial t}+v \frac{\partial \rho}{\partial x}=0
$$

onde é denominada equação linear de advecção (LEVEQUE, 1992, p. 17) ou equação de transporte de massa. Vamos substituir $\rho(x, t)$ por $u=u(x, t)$, onde $u$ designa a concentração de alguma quantidade preservada $Q$, para distinguir o modelo físico $\rho$, do modelo matemático teórico $u$. Seja $f(u)=u V$, isto é, $f$ é uma função de fluxo de massa. Desse modo, para o caso unidimensional, a lei de conservação ou de continuidade dada pela equação (9), fica agora sob a forma conservativa dada por

$$
\frac{\partial u}{\partial t}+\frac{\partial f(u)}{\partial x}=0
$$

Em particular, fazendo $f(u)=\frac{1}{2} u^{2}$ a equação (1) é escrita na forma conservativa da seguinte maneira

$$
\frac{\partial u}{\partial t}+\frac{\partial f(u)}{\partial x}=v \frac{\partial^{2} u}{\partial x^{2}}
$$

A lei de conservação na forma integral (6), nesse mesmo meio unidimensional fica na forma 


$$
\frac{d}{d t} \int_{x_{E}}^{x_{D}} u(x, t) d x+\int_{x_{E}}^{x_{D}} \frac{\partial f(u(x, t))}{\partial x} d x=0
$$

Considere a solução $u=u(x, t)$ e $f(u)$ tal que ambas tenham derivadas contínuas, exceto sobre a curva $x=\xi(t)$ no plano $x t$ onde $u$ tem um salto descontinuo. Sejam $x_{E}$ e $x_{D}$ números reais tais que $x_{E}<x=\xi(t)<x_{D}$, ou seja, um intervalo $\left[x_{E}, x_{D}\right]$ contendo a descontinuidade sendo interpretado como um VC unidimensional. Da continuidade de $u$ temse a existência dos limites laterais dados por

$$
u\left(\xi_{E}, t\right)=\lim _{x \rightarrow \xi(t)^{-}} u(\xi(t), t),
$$

$\mathrm{e}$

$$
u\left(\xi_{D}, t\right)=\lim _{x \rightarrow \xi(t)^{+}} u(\xi(t), t) .
$$

Usando a regra de Leibniz (ÁVILA, 2006, p. 123-124) e o teorema fundamental do cálculo na equação (15) resulta

$$
\int_{x_{E}}^{\xi(t)} \frac{\partial u}{\partial t} d x+u\left(\xi(t)^{-}, t\right) S+\int_{\xi(t)}^{x_{D}} \frac{\partial u}{\partial t} d x-u\left(\xi(t)^{+}, t\right) S=-f\left(u\left(x_{D}, t\right)\right)+f\left(u\left(x_{E}, t\right)\right),
$$

onde

$$
S=\frac{d \xi(t)}{d t}
$$

é denominado velocidade da descontinuidade. Como $\frac{\partial u}{\partial t}$ é limitada, as integrais da equação (18) vão a zero quando $x=\xi(t)$ é aproximado por $x$ respectivamente pela esquerda e pela direita. Daí obtém-se da equação (18) a forma algébrica

$$
\Delta f=S \Delta u
$$

onde $\Delta f=f\left(u\left(x_{D}, t\right)\right)-f\left(u\left(x_{E}, t\right)\right)$ e $\Delta u=u\left(x_{D}, t\right)-u\left(x_{E}, t\right)$. A equação (19) pode ser ainda colocada na forma em que é denominada condição de Hankine-Hugoniot (TORO, 2009, p. 71), dada por

$$
S=\frac{\Delta f}{\Delta u} .
$$

Portanto, para o caso escalar a velocidade de descontinuidade $S$ pode ser resolvida usando as equações (19) e (21). Suponhamos que a equação (1) tenha solução em forma de onda viajante (BIEZUNER, 2010, p. 74), isto é,

$$
u(x, t)=g(x-c t)
$$

onde $c$ é a velocidade da onda, e tal que, para $t=0$ tem-se os limites laterais

$$
u_{E}=\lim _{x \rightarrow \xi(t)^{-}} g(x) \text {, }
$$

$\mathrm{e}$

$$
u_{D}=\lim _{x \rightarrow \xi(t)^{+}} g(x)
$$


Fazendo a mudança de variável $\eta=x-c t$ e usando a equação (14), a equação (1) fica na forma de uma equação diferencial ordinária de segunda ordem dada por

$$
-c \frac{d g(\eta)}{d \eta}+\frac{d\left(f_{o} g\right)(\eta)}{d \eta}=v \frac{d^{2} g(\eta)}{d \eta^{2}},
$$

a qual, resolvida por integração simples resulta na equação diferencial ordinária de primeira ordem na forma

$$
f(g(\eta))-c g(\eta)+A=v \frac{d g(\eta)}{d \eta}
$$

sendo $A$ uma constante de integração. Assumindo que a função $g$ tenda aos valores $u_{E}$ e $u_{D}$ sem oscilações (BIEZUNER, 2010, p. 75), pode-se supor que $\frac{d g(\eta)}{d \eta} \cong 0$ em um pequeno intervalo contendo a descontinuidade em $x=\xi(t)$, ou seja, $g(\eta)$ é aproximadamente constante nesse intervalo e desse modo, obtém-se o sistema linear de ordem dois obtido da eq (26) dado por

$$
\left\{\begin{array}{l}
f\left(u_{E}\right)-c u_{E}+A=0 \\
f\left(u_{D}\right)-c u_{D}+A=0
\end{array}\right.
$$

Do sistema dado pelas equações (27) tem-se que $c=\frac{\Delta f}{\Delta u}$, ou seja, a condição de Rankine-Hugoniot dada pela equação (21). Substituindo o valor de $c$ em qualquer uma das equações (27) pode-se determinar a constante $A$ dada por

$$
A=\frac{u_{D} f\left(u_{D}\right)-u_{E} f\left(u_{E}\right)}{u_{D}-u_{E}} .
$$

A resolução da EDO dada pela equação (26) permite obter $u$ em forma de onda viajante dada pela equação (22). A solução $u=\lim _{v \rightarrow 0} u^{v}(x, t)$ da EDP hiperbólica na forma conservativa dada pela equação (13), onde $u^{v}(x, t)$ é uma solução da equação (14) com $v<1$, é dita solução de viscosidade (BIEZUNER, 2010, p. 73).

\section{Resultados e discussões}

Considerando a equação (1) e a condição inicial (2) e de fronteira (3), e supondo que a solução $u$ é uma função limitada com derivadas necessárias para satisfazer a equação (1), é válida a transformação dada pela equação (4) (COLE, 1951, p. 230). Integrando a equação (4) com respeito a variável $x$ tem-se

$$
-2 v \int_{0}^{x} \frac{\theta_{\xi}(\xi, t)}{\theta(\xi, t)} d \xi=\int_{0}^{x} u(\xi, t) d \xi
$$

A integral do lado esquerdo da equação (29) tem como uma primitiva a função $\ln \theta(\xi, t)$ . Daí, e do teorema fundamental do cálculo resulta

$$
\theta(x, t)=\theta(0, t) \exp \left[-\frac{1}{2 v} \int_{0}^{x} u_{0}(\xi, t) d \xi\right],
$$


onde $u_{0}(x)=u(x, 0)$. Em particular

$$
\theta_{0}(x)=\theta(x, 0)=C_{0} \exp \left[-\frac{1}{2 v} \int_{0}^{x} u_{0}(\xi) d \xi\right]
$$

sendo $C_{0}=\theta(0,0)$ uma constante que será cancelada pela transformação de Cole. Com o objetivo de apresentarmos outras representações da solução da equação de calor não disponibilizadas por Cole (1951, p. 231-235) e também por Benton e Platzman (1972, p. 202208), vamos analisar com mais detalhes no que diz respeito à obtenção da solução, o primeiro exemplo da equação de Burgers (1) em um meio infinito unidimensional com condição inicial dada por uma função constante descontinua por partes, apresentado por Cole (1951, p. 232). Esse exemplo trata-se, ainda segundo Cole, de uma onda de choque que se aproxima rapidamente do estado estacionário. As condições iniciais são

$$
u_{0}(x)=\left\{\begin{array}{cc}
u_{1} & x<0 \\
-u_{1} & x>0
\end{array}\right.
$$

Da equação (31) tem-se

$$
\theta_{0}(x)=\left\{\begin{array}{cc}
C_{0} \exp \left(\frac{u_{1} x}{2 v}\right) & x>0, \\
C_{0} \exp \left(-\frac{u_{1} x}{2 v}\right) & x<0 .
\end{array}\right.
$$

A solução para a equação de calor (5) em um meio infinito com condição inicial $\theta_{0}(x)$ é dada segundo Carslaw e Jaeger (1959, p. 53) por

$$
\theta(x, t)=\frac{1}{2 \sqrt{\pi v t}} \int_{-\infty}^{\infty} \exp \left[-\frac{(x-\xi)^{2}}{4 v t}\right] \theta_{0}(\xi) d \xi .
$$

Das equações (33) e (34) tem-se

$$
\theta(x, t)=\frac{C_{0}}{2 \sqrt{\pi v t}}\left\{\int_{-\infty}^{0} \exp \left[-\frac{(x-\xi)^{2}}{4 v t}\right] \exp \left(-\frac{u_{1} \xi}{2 v}\right) d \xi+\int_{0}^{\infty} \exp \left[-\frac{(x-\xi)^{2}}{4 v t}\right] \exp \left(\frac{u_{1} \xi}{2 v}\right) d \xi\right\}
$$

Vamos resolver a segunda integral da equação (35) de modo que a primeira terá solução análoga.

$$
\begin{aligned}
\int_{0}^{\infty} \exp \left[-\frac{(x-\xi)^{2}}{4 v t}\right] \exp \left(\frac{u_{1} \xi}{2 v}\right) d \xi & =\exp \left(-\frac{x^{2}}{4 v t}\right) \lim _{M \rightarrow \infty} \int_{0}^{M} \exp \left[-\frac{\left(\xi^{2}-2 \xi\left(x+u_{1} t\right)+\left(x+u_{1} t\right)^{2}\right)}{4 v t}\right] \exp \left(-\frac{\left(x+u_{1} t\right)^{2}}{4 v t}\right) d \xi= \\
& =\exp \left(\frac{\left(2 x+u_{1} t\right) u_{1}}{4 v}\right) \lim _{M \rightarrow \infty} \int_{0}^{M} \exp \left[-\frac{\left(\xi-\left(x+u_{1} t\right)^{2}\right)}{4 v t}\right] d \xi
\end{aligned}
$$

Fazendo a mudança de variável $\omega=\frac{\xi-\left(x+u_{1} t\right)}{2 \sqrt{v t}}$ na integral (36) resulta 


$$
\int_{0}^{\infty} \exp \left[-\frac{(x-\xi)^{2}}{4 v t}\right] \exp \left(\frac{u_{1} \xi}{2 v}\right) d \xi=2 \sqrt{v t} \exp \left(\frac{\left.\left(2 x+u_{1} t\right) u_{1}\right)}{4 v}\right)\left[\int_{-\frac{x+u_{1} t}{2 \sqrt{v t}}}^{0} \mathrm{e}^{-\omega^{2}} d \omega+\lim _{M \rightarrow \infty} \int_{0}^{\frac{M-x+u_{1} t}{2 \sqrt{v t}}} \mathrm{e}^{-\omega^{2}} d \omega\right]
$$

ou seja,

$$
\int_{0}^{\infty} \exp \left[-\frac{(x-\xi)^{2}}{4 v t}\right] \exp \left(\frac{u_{1} \xi}{2 v}\right) d \xi=2 \sqrt{v t} \exp \left(\frac{\left(2 x+u_{1} t\right) u_{1}}{4 v}\right)\left[1+\operatorname{erf}\left(\frac{x+u_{1} t}{2 \sqrt{v t}}\right)\right],
$$

onde $\operatorname{erf}(x)=\frac{2}{\sqrt{\pi}} \int_{0}^{x} \exp \left(-\eta^{2}\right) d \eta$, é a função erro de Gauss satisfazendo as seguintes propriedades (CARSLAW; JAEGER, 1959, p. 51)

$$
\operatorname{erf}(0)=0, \operatorname{erf}(\infty)=1, \quad \operatorname{erf}(-x)=-\operatorname{erf}(x) \text { e } \operatorname{erf}_{C}(x)=1-\operatorname{erf}(x) .
$$

De modo análogo obtém-se

$$
\int_{-\infty}^{0} \exp \left[-\frac{(x-\xi)^{2}}{4 v t}\right] \exp \left(\frac{u_{1} \xi}{2 v}\right) d \xi=2 \sqrt{v t} \exp \left(\frac{\left(-2 x+u_{1} t\right) u_{1}}{4 v}\right)\left[1+\operatorname{erf}\left(\frac{-x+u_{1} t}{2 \sqrt{v t}}\right)\right] \text {. }
$$

Das equações (35), (38) e (40) tem-se a solução a equação de calor dado pelas equações (5) e (33) na forma

$$
\theta(x, t)=\frac{C_{0}}{2} \mathrm{e}^{\frac{u_{1}^{2} t}{4 v}}\left[\mathrm{e}^{\frac{-u_{1} x}{2 v}}+\mathrm{e}^{\frac{u_{1} x}{2 v}}+\mathrm{e}^{\frac{-u_{1} x}{2 v}} \operatorname{erf}\left(\frac{-x+u_{1} t}{2 \sqrt{v t}}\right)+\mathrm{e}^{\frac{u_{1} x}{2 v}} \operatorname{erf}\left(\frac{x+u_{1} t}{2 \sqrt{v t}}\right)\right],
$$

ou ainda, como

$$
\theta(x, t)=\frac{1}{2} C_{0} \mathrm{e}^{\frac{u_{1}^{2} t}{4 v}}\left[2 \cosh \left(\frac{u_{1} x}{2 v}\right)+\mathrm{e}^{\frac{u_{1} x}{2 v}} \operatorname{erf}\left(\frac{x+u_{1} t}{2 \sqrt{v t}}\right)-\mathrm{e}^{\frac{-u_{1} x}{2 v}} \operatorname{erf}\left(\frac{-x+u_{1} t}{2 \sqrt{v t}}\right)\right] .
$$

Daí, e usando o fato que

$$
\frac{\partial}{\partial x} \operatorname{erf}\left(\frac{x}{2 \sqrt{v t}}\right)=\frac{1}{\sqrt{\pi v t}} \exp \left(\frac{-x^{2}}{4 v t}\right)
$$

tem-se

$$
\frac{\partial}{\partial x} \theta(x, t)=\frac{C_{0}}{4 v} \mathrm{e}^{\frac{u_{1}^{2} t}{4 v}}\left[\begin{array}{l}
\frac{2 u_{1}}{v} \operatorname{senh}\left(\frac{u_{1} x}{2 v}\right)+\mathrm{e}^{\frac{u_{1} x}{2 v}}\left(\frac{u_{1}}{v} \operatorname{erf}\left(\frac{x+u_{1} t}{2 \sqrt{v t}}\right)+\frac{2 \mathrm{e}^{\frac{-\left(u_{1}+x\right)^{2}}{4 v t}}}{\sqrt{\pi v t}}\right)+ \\
-\mathrm{e}^{\frac{-u_{1} x}{2 v}}\left(\frac{u_{1}}{v} \operatorname{erf}\left(\frac{u_{1} t-x}{2 \sqrt{v t}}\right)+\frac{2 \mathrm{e}^{\frac{-\left(t u_{1}-x\right)^{2}}{4 v t}}}{\sqrt{\pi v t}}\right) \operatorname{erf}\left(\frac{x-u_{1} t}{2 \sqrt{v t}}\right)
\end{array}\right] .
$$

Das equações (4), (42) e (44) resulta a solução explícita para a equação (1) com as condições (32) dada por 


$$
u(x, t)=-u_{1} \frac{2 \mathrm{e}^{\frac{u_{1} x}{2 v}} \operatorname{senh}\left(\frac{u_{1} x}{2 v}\right)+\mathrm{e}^{\frac{u_{1} x}{v}} \operatorname{erf}\left(\frac{x+u_{1} t}{2 \sqrt{v t}}\right)+\operatorname{erf}\left(\frac{x-u_{1} t}{2 \sqrt{v t}}\right)}{2 \mathrm{e}^{\frac{u_{1} x}{2 v}} \cosh \left(\frac{u_{1} x}{2 v}\right)+\mathrm{e}^{\frac{u_{1} x}{v}} \operatorname{erf}\left(\frac{x+u_{1} t}{2 \sqrt{v t}}\right)-\operatorname{erf}\left(\frac{x-u_{1} t}{2 \sqrt{v t}}\right)} .
$$

Quando $t \rightarrow 0$ as condições iniciais dadas pela equação (32) são válidas, usando para isso as propriedades $\operatorname{erf}( \pm \infty)= \pm 1$. Note que a constante $C_{0}$ foi eliminada na transformação de Cole, conforme comentado anteriormente.

A Fig. 1 mostra o perfil da solução da equação de Burgers dado pela equação (45) para $u_{1}=1, v=1.0,0.1 \mathrm{e} 0.01$ no tempo $t=0.02$.

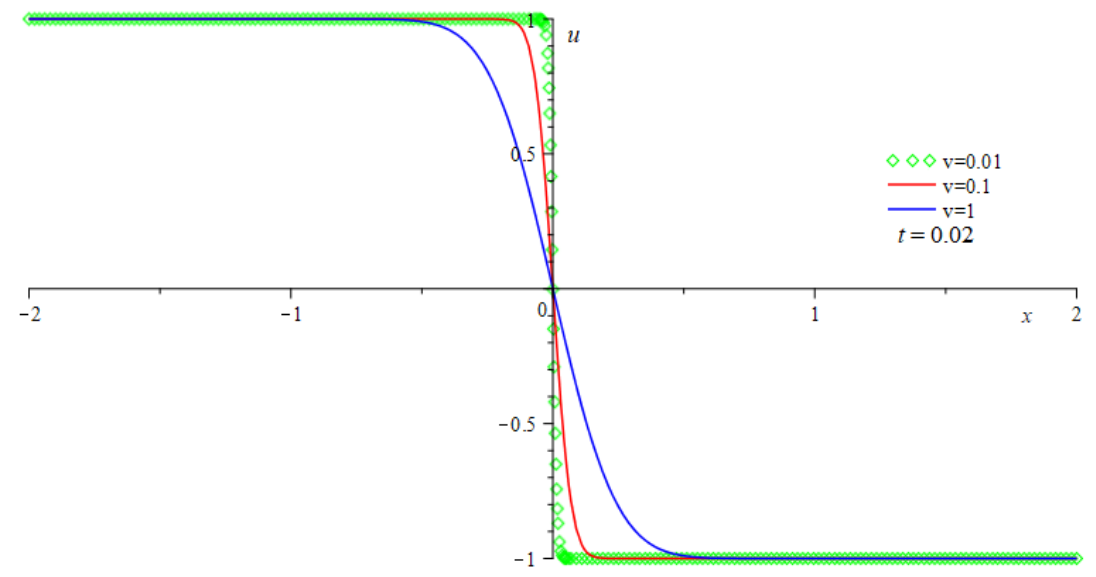

Figura 1: Perfil da solução da equação de Burgers em meio infinito.

Pode ser notado que para $v<1$ a descontinuidade inicial não é suavizada pelo termo parabólico na equação (1).

Com base nesse problema, outras condições iniciais constantes por partes podem ser estabelecidas em um meio infinito unidimensional, bem como, suas soluções explícitas.

\section{I) Se as condições iniciais forem}

$$
u_{0}(x)= \begin{cases}u_{1} & |x|<L \\ 0 & |x| \geq L\end{cases}
$$

Tem-se das equações $(31,46)$

$$
\theta_{0}(x)=\left\{\begin{array}{cc}
C_{o} \exp \left(-\frac{u_{1} x}{2 v}\right) u_{1} & |x|<L \\
C_{o} & |x| \geq L
\end{array}\right.
$$

Das equações (34) e (47) resulta

$$
\theta(x, t)=\frac{C_{0}}{2 \sqrt{\pi v t}}\left\{\int_{-\infty}^{-L} \mathrm{e}^{-\frac{(x-\xi)^{2}}{4 v t}} d \xi+\int_{-L}^{L} \mathrm{e}^{-\frac{(x-\xi)^{2}}{4 v t}} \mathrm{e}^{-\frac{u_{1} \xi}{2 v}} d \xi+\int_{L}^{\infty} \mathrm{e}^{-\frac{(x-\xi)^{2}}{4 v t}} d \xi\right\} .
$$

Fazendo a mudança de variável 


$$
\beta=\frac{x-\xi}{2 \sqrt{v t}}
$$

obtém-se

$$
\theta(x, t)=\frac{C_{0}}{2}\left\{\operatorname{erf}_{C}\left(\frac{x+L}{2 \sqrt{v t}}\right)+\operatorname{erf}_{C}\left(\frac{L-x}{2 \sqrt{v t}}\right)+\mathrm{e}^{\frac{u_{1}\left(u_{1} t-2 x\right)}{4 v}}\left[\operatorname{erf}\left(\frac{L+x-u_{1} t}{2 \sqrt{v t}}\right)+\operatorname{erf}\left(\frac{L-x+u_{1} t}{2 \sqrt{v t}}\right)\right]\right\}
$$

Quando $t \rightarrow 0$ as condições iniciais dadas pela equação (47) são válidas, usando para isso as propriedades $\operatorname{erf}( \pm \infty)= \pm 1$ e analisando a posição do $x$ com respeito à $L$. Das equações (4) e (49) tem-se

$$
u(x, t)=\frac{-2 v \theta_{x}(x, t)}{\theta(x, t)}
$$

ou, de modo simplificado,

$$
u(x, t)=\frac{A(x, t)}{B(x, t)}
$$

onde

$$
\begin{gathered}
A(x, t)=-u_{1} \sqrt{\pi v t} e^{\frac{u_{1}\left(u_{1} t-2 x\right)}{4 v}}\left(\operatorname{erf}\left(\frac{L+x-t u_{1}}{2 \sqrt{v t}}\right)+\operatorname{erf}\left(\frac{L-x+t u_{1}}{2 \sqrt{v t}}\right)\right)+ \\
+4 v e^{\frac{-\left(L^{2}+x^{2}\right)}{4 v t}}\left(\operatorname{senh}\left(\frac{L\left(u_{1} t-x\right)}{2 v t}\right)-\operatorname{senh}\left(\frac{x L}{2 v t}\right)\right)
\end{gathered}
$$

$\mathrm{e}$

$$
B(x, t)=-\sqrt{\pi v t}\left[e^{\frac{u_{1}\left(u_{1} t-2 x\right)}{4 v}}\left(\operatorname{erf}\left(\frac{L+x-t u_{1}}{2 \sqrt{v t}}\right)+\operatorname{erf}\left(\frac{L-x+t u_{1}}{2 \sqrt{v t}}\right)\right)-\operatorname{erf}_{c}\left(\frac{L+x}{2 \sqrt{v t}}\right)-\operatorname{erf}\left(\frac{L-x}{2 \sqrt{v t}}\right)\right]
$$

A Fig.2 mostra o gráfico de $u(x, t)$ para $u_{1}=1, v=1.0,0.1$ e 0.01 no tempo $t=0.02$.

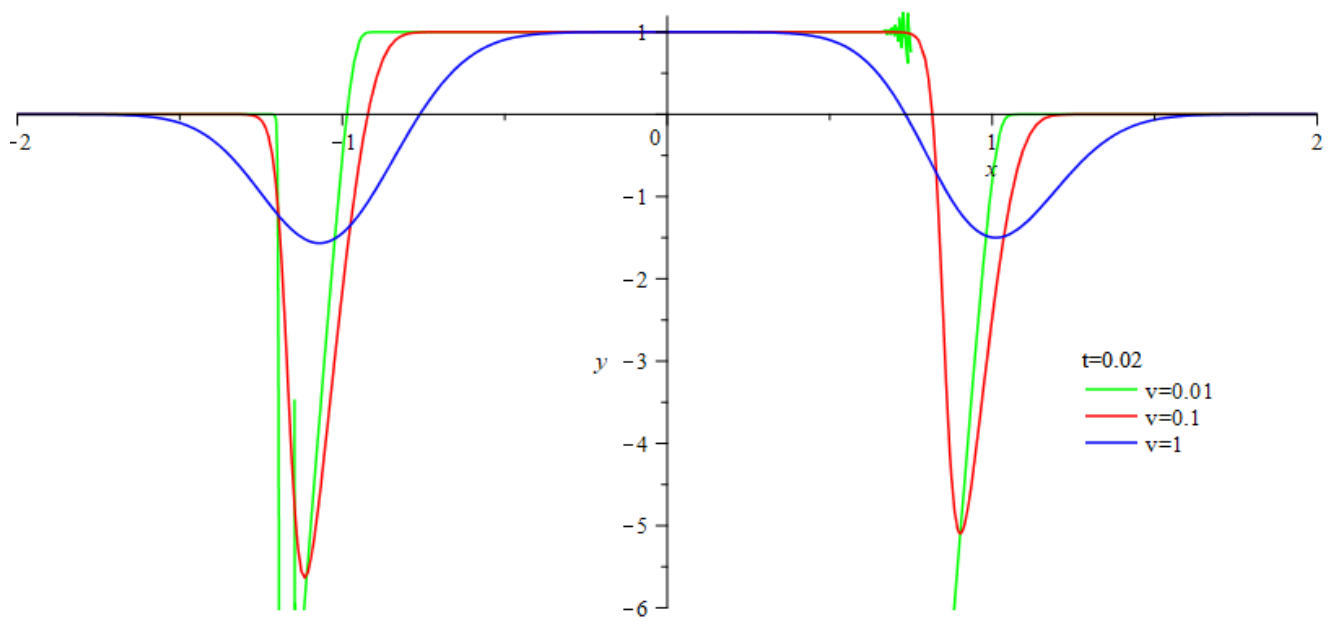

Figura 2: Gráfico de $u(x, t=0.02)$, considerando $u_{1}=1 \mathrm{e} L=1$.

Quando $t \rightarrow 0$ as condições iniciais dadas pela equação (46) são válidas, usando para isso as propriedades $\operatorname{erf}( \pm \infty)= \pm 1$ e analisando a posição do $x$ com respeito à $L$. 
Pode ser notado que à medida que $v \rightarrow 0$ a equação (1) tende a uma equação de Burgers inviscida do tipo hiperbólica, onde as descontinuidades são transportas sem suavização, podendo haver nesse caso, a formação de choques ou singularidades que podem trazer dificuldades para o seu tratamento numérico (CUMINATO; MENEGUETTE JUNIOR, 2013, p. 228). Note que quando $v=1$, o termo parabólico da equação (1) suaviza a descontinuidade dos dados iniciais.

\section{II) Se as condições iniciais forem}

$$
u_{0}(x)= \begin{cases}0 & |x|<L \\ u_{1} & |x| \geq L\end{cases}
$$

Tem-se das equações (31) e (55)

$$
\theta_{0}(x)= \begin{cases}C_{0} & |x|<L \\ C_{0} \exp \left(-\frac{u_{1} x}{2 v}\right) & |x| \geq L\end{cases}
$$

Das equações (34) e (56) tem-se

$$
\theta(x, t)=\frac{C_{0}}{2 \sqrt{\pi v t}}\left\{\int_{-\infty}^{L} \mathrm{e}^{-\frac{(x-\xi)^{2}}{4 v t}} \mathrm{e}^{-\frac{u_{1 \xi}}{2 v}} d \xi+\int_{-L}^{L} \mathrm{e}^{-\frac{(x-\xi)^{2}}{4 v t}} d \xi+\int_{L}^{\infty} \mathrm{e}^{-\frac{(x-\xi)^{2}}{4 v t}} \mathrm{e}^{-\frac{u_{1} \xi}{2 v}} d \xi\right\} .
$$

Usando a equação (49) pode-se obter

$$
\theta(x, t)=\frac{C_{0}}{2}\left[\operatorname{erf}\left(\frac{L-x}{2 \sqrt{v t}}\right)+\operatorname{erf}\left(\frac{L+x}{2 \sqrt{v t}}\right)+\mathrm{e}^{\frac{u_{1}\left(-2 x+u_{1} t\right)}{4 v}}\left(\operatorname{erf}_{c}\left(\frac{L+x-u_{1} t}{2 \sqrt{v t}}\right)+\operatorname{erf}_{c}\left(\frac{L-x+u_{1} t}{2 \sqrt{v t}}\right)\right)\right]
$$

Da equação (58) e usando a equação (4) tem-se

$$
u(x, t)=\frac{A(x, t)}{B(x, t)}
$$

onde

$$
A(x, t)=\left\{\begin{array}{l}
2 v \mathrm{e}^{-\frac{(L+x)^{2}}{4 v t}}-2 v \mathrm{e}^{-\frac{(L-x)^{2}}{4 v t}}-\sqrt{\pi v t} u_{1} \mathrm{e}^{\frac{u_{1}\left(-2 x+u_{1} t\right)}{4 v t}}\left(\operatorname{erf}\left(\frac{L-x+u_{1} t}{2 \sqrt{v t}}\right)+\operatorname{erf}\left(\frac{L+x-u_{1} t}{2 \sqrt{v t}}\right)\right) \\
-2 v \mathrm{e}^{-\frac{x^{2}+L^{2}-2 x L+2 u_{1} L t}{4 v t}}+2 v \mathrm{e}^{-\frac{x^{2}+L^{2}+2 x L-2 u_{1} L t}{4 v t}}
\end{array}\right\}
$$

$\mathrm{e}$

$$
B(x, t)=\sqrt{\pi v t}\left\{\begin{array}{l}
\exp \left[\frac{u_{1}\left(-2 x+u_{1} t\right)}{4 v t}\right]\left[\operatorname{erf}\left(\frac{L-x+u_{1} t}{2 \sqrt{v t}}\right)+\operatorname{erf}\left(\frac{L+x-u_{1} t}{2 \sqrt{v t}}\right)\right]+ \\
-\operatorname{erf}\left(\frac{L+x}{2 \sqrt{v t}}\right)-\operatorname{erf}\left(\frac{L-x}{2 \sqrt{v t}}\right)-2 \exp \left[\frac{u_{1}\left(-2 x+u_{1} t\right)}{4 v t}\right]
\end{array}\right\} .
$$

A Fig.3 mostra o gráfico de $u(x, t)$ para diversos valores de $v$. 


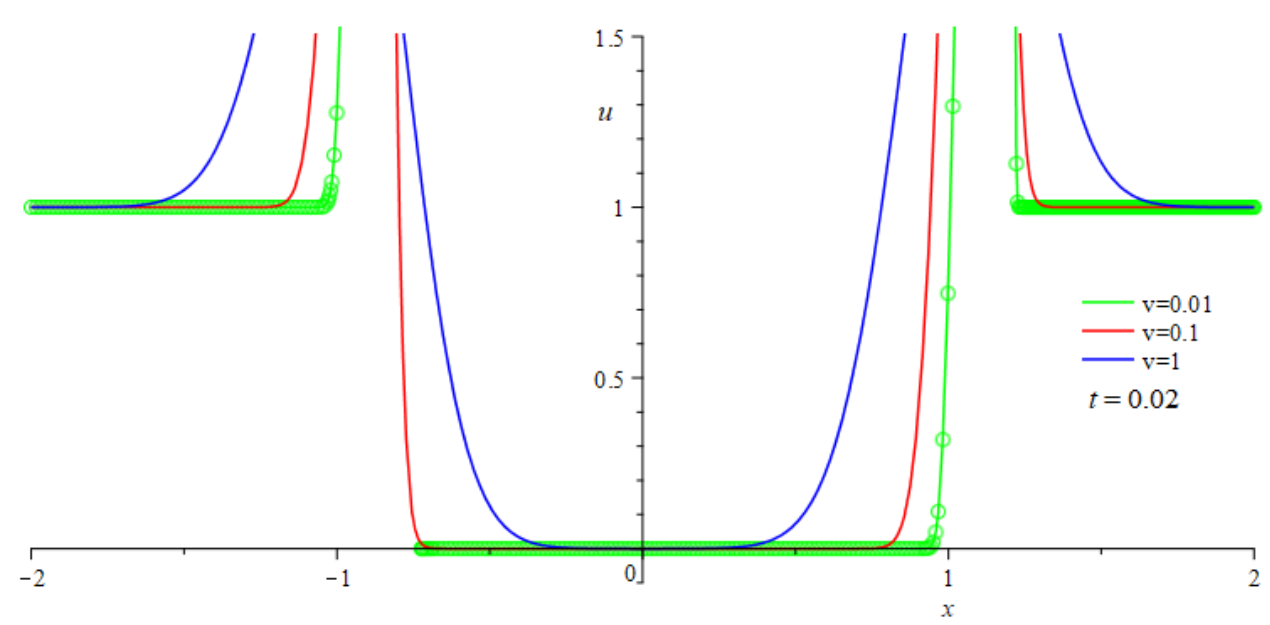

Figura 3: Gráfico de $u(x, t=0.02)$, considerando $u_{1}=1$ e $L=1$.

\section{III) Se as condições iniciais forem}

$$
u(x, 0)=u_{0}(x), \quad x>0
$$

e a condição de fronteira do tipo

$$
u(0, t)=0, \quad t>0,
$$

tem-se das equações (4), (30) e (31) o problema de Cauchy representado pela equação de calor dada por

$$
\begin{gathered}
\left(\frac{\partial \theta}{\partial t}\right)=v\left(\frac{\partial^{2} \theta}{\partial x^{2}}\right), \quad x>0, \quad t>0, \\
\theta(x, 0)=\theta_{0}(x), \quad x \geq 0, \\
\frac{\partial \theta}{\partial x}(0, t)=0, \quad t>0 .
\end{gathered}
$$

A condição de fronteira dada pela equação (66) indica que não há fluxo de calor em $x=0$. Esse problema tem solução única, e é dada segundo Carslaw e Jaeger (1959, p. 56) por

$$
\theta(x, t)=\frac{1}{2 \sqrt{\pi v t}} \int_{0}^{\infty}\left(\exp \left[-\frac{(x-\xi)^{2}}{4 v t}\right]-\exp \left[-\frac{(x+\xi)^{2}}{4 v t}\right]\right) \theta_{0}(\xi) d \xi
$$

Em particular, se $u_{0}(x)=u_{1}$, resulta da equação (31)

$$
\theta_{0}(x)=C_{0} \exp \left(-\frac{u_{1} x}{2 v}\right)
$$

que substituído na equação (67) obtém-se 


$$
\theta(x, t)=\frac{1}{2 \sqrt{\pi v t}} \int_{0}^{\infty}\left(\exp \left[-\frac{(x-\xi)^{2}}{4 v t}\right]-\exp \left[-\frac{(x+\xi)^{2}}{4 v t}\right]\right) \exp \left(-\frac{u_{1} \xi}{2 v}\right) d \xi
$$

As integrais que aparecem na equação (69) já foram tratadas nos casos anteriores fazendo para isso a mudança de variável $\beta_{1}=\frac{x-\xi}{2 \sqrt{v t}}$ para a primeira integral imprópria e $\beta_{2}=\frac{x+\xi}{2 \sqrt{v t}}$ para a segunda. Com isso, a solução para o problema de Cauchy dado pelas equações (64-66) é dada por

$$
\theta(x, t)=\frac{1}{2} C_{0} \mathrm{e}^{\frac{u_{1}^{2} t}{4 v}}\left[2 \cosh \left(\frac{u_{1} x}{2 v}\right)+\mathrm{e}^{-\frac{u_{1} x}{2 v}} \operatorname{erf}\left(\frac{x-u_{1} t}{2 \sqrt{v t}}\right)-\mathrm{e}^{\frac{u_{1} x}{2 v}}+\operatorname{erf}\left(\frac{x+u_{1} t}{2 \sqrt{v t}}\right)\right],
$$

Muito similar a equação (42), o que era de se esperar, pois o caso semi-infinito pode ser tratado como um caso particular do meio infinito. Das equações (4) e (70) obtemos a solução para a equação (1) com as condições (62) e (63), dada por

$$
u(x, t)=-u_{1} \frac{2 \operatorname{senh}\left(\frac{u_{1} x}{2 v}\right)-\left\{\exp \left(\frac{u_{1} x}{2 v}\right) \operatorname{erf}\left(\frac{x+u_{1} t}{2 \sqrt{v t}}\right)+\exp \left(-\frac{u_{1} x}{2 v}\right)+\operatorname{erf}\left(\frac{x-u_{1} t}{2 \sqrt{v t}}\right)\right\}}{2 \cosh \left(\frac{u_{1} x}{2 v}\right)-\left\{\exp \left(\frac{u_{1} x}{2 v}\right) \operatorname{erf}\left(\frac{x+u_{1} t}{2 \sqrt{v t}}\right)-\exp \left(-\frac{u_{1} x}{2 v}\right)+\operatorname{erf}\left(\frac{x-u_{1} t}{2 \sqrt{v t}}\right)\right\}},
$$

cuja solução é similar a solução dada pela equação (45) do problema em meio infinito com condições iniciais constante por partes resolvido por Cole (1951, p. 232). Quando $t \rightarrow 0 \mathrm{a}$ condição inicial dada pela equação (68) é válida. Para ver isso basta usar as propriedades $\operatorname{erf}( \pm \infty)= \pm 1$. De fato, se $t \rightarrow 0$ tem-se da equação (71) que

$$
u(x, 0)=-u_{1} \frac{2 \operatorname{senh}\left(\frac{u_{1} x}{2 v}\right)-2 \cosh \left(\frac{u_{1} x}{2 v}\right)}{2 \cosh \left(\frac{u_{1} x}{2 v}\right)-2 \operatorname{senh}\left(\frac{u_{1} x}{2 v}\right)}=u_{1},
$$

e para verificar a equação (63) basta utilizar diretamente a equação (71) para obter

$$
u(0, t)=-u_{1} \frac{2 \operatorname{senh}(0)}{2 \operatorname{erf}\left(\frac{u_{1} t}{2 \sqrt{v t}}\right)}=0 .
$$

A Fig. 4 mostra o perfil da solução dada pela equação (71) para $u_{1}=1$ e $v=1$ nos tempos $t=0,3,0.4,0.5 e 0.6$. 


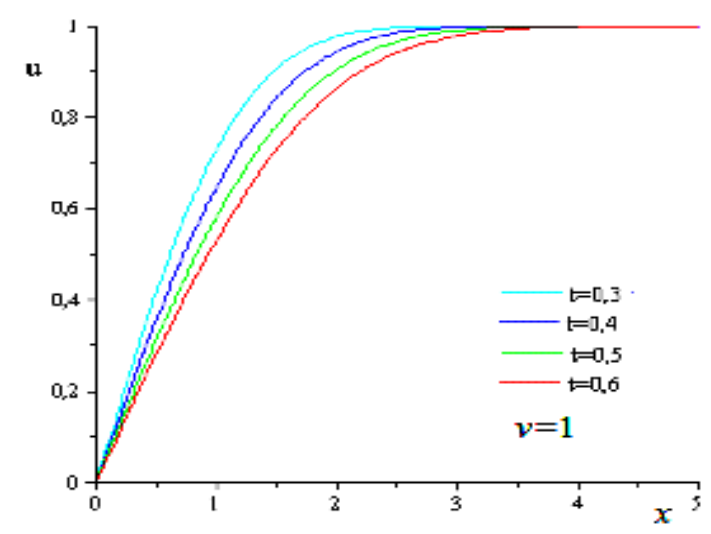

Figura 4. Perfil da solução no meio semi-infinito nos tempos $t=0.3,0.4,0.5 e 0.6$.

\section{IV- A condição inicial e de fronteira são dadas por}

$$
\begin{array}{ll}
u(x, 0)=u_{0}(x), & 0 \leq x \leq L, \\
u(0, t)=u(L, t)=0, & t>0 .
\end{array}
$$

Tais condições correspondem, segundo Cole (1951, p. 233), a um modelo teórico de decaimento de uma turbulência arbitrária inicial confinada a uma caixa. A solução exata desse problema (COLE, 1951, p. 233) para a condição inicial ou perturbação periódica dada por $u=u_{0} \operatorname{sen}\left(\frac{\pi x}{L}\right)$, foi utilizada por vários pesquisadores, como por exemplo: Caldwell e Smith (1982), Dhawan, Kapoor, Kumar e Rawat (2012) Hon e Mao (1998), Kutluay, Bahadir e Özdeş (1999), Mittal e Singhal (1993) e Romao, Martins e Moura (2013) para resolverem a equação de Burgers numericamente utilizando variadas técnicas. Devido à importância teórica desse modelo, vamos descrever mais detalhadamente a solução desse problema e apresentar mais um modelo periódico de turbulência inicial para uma comparação com o modelo proposto por Cole. por

Das equações (4) e (31) e das condições (74) e (75) tem-se a EDP linear de calor dada

$$
\begin{aligned}
& \left(\frac{\partial \theta}{\partial t}\right)=v\left(\frac{\partial^{2} \theta}{\partial x^{2}}\right), \quad x>0, \quad t>0, \\
& \theta_{0}(x)=C_{0} \exp \left(-\frac{1}{2 v} \int_{0}^{x} u_{0}(\xi) d \xi\right) \quad x \geq 0 \\
& \frac{\partial \theta}{\partial x}(0, t)=\frac{\partial \theta}{\partial x}(L, t), \quad t>0 .
\end{aligned}
$$

Esse problema tem solução única e limitada, e pode ser obtida pelo método de separação de variáveis sendo dada segundo Boyce e DiPrima (1999, p. 413) por

$$
\theta(x, t)=A_{0}+\sum_{n=1}^{\infty} \exp \left(-\frac{v n^{2} \pi^{2} t}{L^{2}}\right) A_{n} \cos \left(\frac{n \pi x}{L}\right),
$$


onde os coeficientes $A_{0}$ e $A_{n}$ são determinados pela condição inicial, isto é,

$$
\theta_{0}(x)=A_{0}+\sum_{n=1}^{\infty} A_{n} \cos \left(\frac{n \pi x}{L}\right)
$$

Cole (1951, p. 233) não mostrou como foram determinados esses coeficientes, o que faremos a seguir. Multiplicando ambos os lados da equação (80) por $\cos \left(\frac{n \pi x}{L}\right)$ e integrando de 0 a $L$ e usando as relações

$$
\int_{0}^{L} \cos \left(\frac{p \pi x}{L}\right) \cos \left(\frac{n \pi x}{L}\right) d x=\left\{\begin{array}{ll}
0 & p \neq n \\
\frac{L}{2} & p=n
\end{array},\right.
$$

obtemos as seguintes relações

$$
\begin{aligned}
& \int_{0}^{L} \theta_{0}(x) \cos \left(\frac{n \pi x}{L}\right) d x=A_{n} \int_{0}^{L} \cos ^{2}\left(\frac{n \pi x}{L}\right) d x=A_{0} L, \\
& \int_{0}^{L} \theta_{0}(x) \cos \left(\frac{n \pi x}{L}\right) d x=A_{n} \int_{0}^{L} \cos ^{2}\left(\frac{n \pi x}{L}\right) d x=A_{n} \frac{L}{2} .
\end{aligned}
$$

Das equações (82) e (83) e da equação (31) tem-se

$$
A_{0}=\frac{1}{L} C_{0} \int_{0}^{L} \exp \left(-(2 v)^{-1} \int_{0}^{x} u_{0}(\xi) d \xi\right) d x,
$$

e para $n>0$

$$
A_{n}=\frac{2}{L} C_{0} \int_{0}^{L} \exp \left(-(2 v)^{-1} \int_{0}^{x} u_{0}(\xi) d \xi\right) \cos \left(\frac{n \pi x}{L}\right) d x .
$$

Das equações (79), (84) e (85) e da transformação dada pela equação (4) resulta

$$
u(x, t)=\frac{2 \pi v}{L} \frac{\sum_{n=1}^{\infty} n \exp \left(-\frac{v n^{2} \pi^{2} t}{L^{2}}\right) A_{n} \operatorname{sen}\left(\frac{n \pi x}{L}\right)}{A_{0}+\sum_{n=1}^{\infty} \exp \left(-\frac{v n^{2} \pi^{2} t}{L^{2}}\right) A_{n} \cos \left(\frac{n \pi x}{L}\right)} .
$$

Vale ressaltar que no artigo original de Cole (1951, p. 233) os termos $A_{n}$ não estavam corretamente apresentados, pois faltava na equação (45b) do tex to citado, o termo em cosseno como aparece na equação (85) onde atribuímos esse fato a um possível erro de digitação. Para largos tempos $t$ Cole (1951, p. 234) derivou uma equação mais simples para $u(x, t)$ considerando só o primeiro termo do numerador $A_{1}$ para obter a equação aproximada dada por

$$
u(x, t) \cong \frac{2 \pi v}{L} \frac{A_{1}}{A_{0}} \exp \left(-\frac{v \pi^{2} t}{L^{2}}\right) \operatorname{sen}\left(\frac{\pi x}{L}\right) .
$$


Para $u_{0}(x)=u_{0} \operatorname{sen}\left(\frac{\pi x}{L}\right) \operatorname{com} u_{0}=1$ e $L=1$, a solução dada pela equação (86) fica na forma

$$
u(x, t)=2 \pi v \frac{\sum_{n=1}^{\infty} n \exp \left(-v n^{2} \pi^{2} t\right) A_{n} \operatorname{sen}(n \pi x)}{A_{0}+\sum_{n=1}^{\infty} \exp \left(-v n^{2} \pi^{2} t\right) A_{n} \cos (n \pi x)}
$$

onde

$$
\begin{aligned}
& A_{0}=C_{0} \int_{0}^{1} \exp \left\{-\frac{[1-\cos (\pi x)]}{2 v}\right\} d x, \\
& A_{n}=2 C_{0} \int_{0}^{1} \exp \left\{-\frac{[1-\cos (\pi x)]}{2 v}\right\} \cos (n \pi x) d x .
\end{aligned}
$$

Para $u_{0}(x)=u_{0} \cos \left(\frac{\pi x}{L}\right) \operatorname{com} u_{0}=1$ e $L=1$, tem-se

$$
\begin{aligned}
& A_{0}=C_{0} \int_{0}^{1} \exp \left[-(2 v)^{-1} \operatorname{sen}(\pi x)\right] d x, \\
& A_{n}=2 C_{0} \int_{0}^{1} \exp \left[-(2 v)^{-1} \operatorname{sen}(\pi x)\right] \cos (n \pi x) d x .
\end{aligned}
$$

A Fig.5a mostra o perfil da solução para $u_{0}(x)=\operatorname{sen}(\pi x)$ e a Fig.5b, mostra o perfil da solução quando $u_{0}(x)=\cos (\pi x)$ com $R=20$ ou $v=0.05$ para os instantes $t=0.01,0.4,0,5$ e 0.6 .

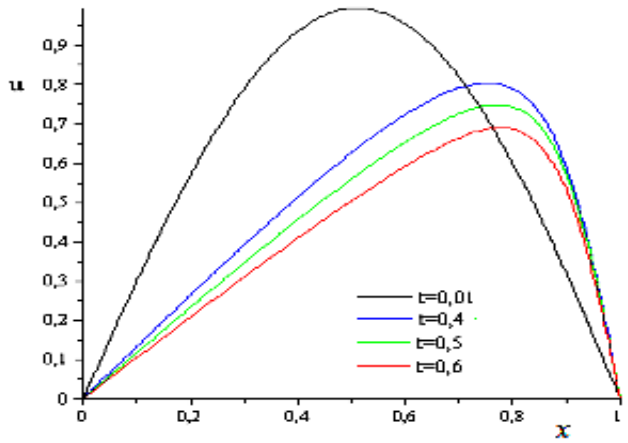

a)

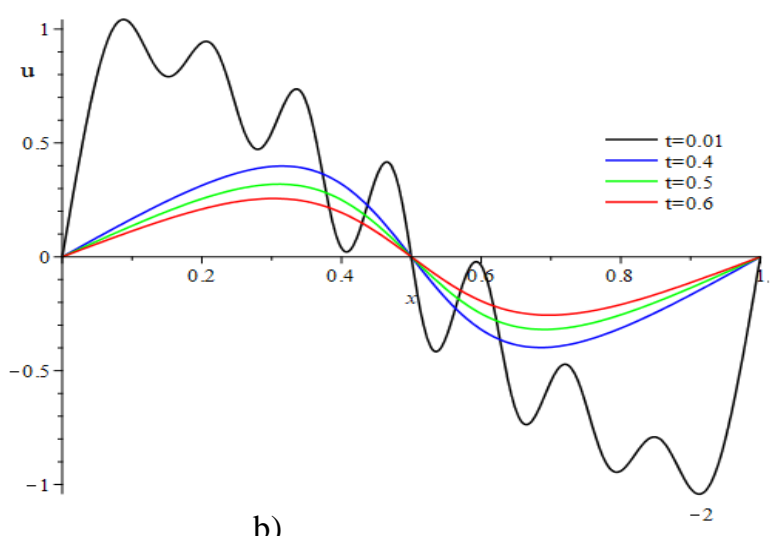

b)

Figura 5: Curvas soluções da equação de Burgers para $R=20$.

A Fig. 5(a) mostra a dominância do termo convectivo sobre o termo difusivo com a solução $u$ formando uma onda de choque à direita com velocidade proporcional a $u$. Esse perfil 
de $u$ é concordante com a Fig.1c obtida por Hon e Mao (1998, p. 46) para $R=10$. Na Fig.5(b) é possível notar que a difusão é dominante, havendo pouca contribuição do termo convectivo para o balanceamento da solução da equação de Burgers sendo talvez esse um dos motivos desse exemplo não figurar na aplicação de métodos numéricos das referências anteriormente citadas sobre a resolução numérica da equação de Burgers. É possível observar uma maior rapidez no decaimento da turbulência em relação à perturbação inicial conforme mostrado na Fig.6. O uso da equação (88) é limitado, ou seja, segundo Hon e Mao (1998, p. 41) para valores de $R \geq 4500$ não é possível computa-la, sendo que até mesmo para valores $R \geq 10$ a convergência da equação (88) pode ser lenta (CALDWELL; SMITH, 1982, p. 382).

\section{V-A condição inicial é dada por}

$$
u_{0}(x)= \begin{cases}1 & x<0 \\ 0 & x>0\end{cases}
$$

Usando a equação (14) onde $f(u)=\frac{u^{2}}{2}$, a equação (1) fica na forma conservativa não homogênea, isto é, não são desprezados os efeitos dissipativos devido à viscosidade do fluído. Das equações (22) e (26) tem-se a EDO linear de Bernoulli dada por

$$
\frac{d \omega}{d \eta}-\frac{\omega}{2 v}=-\frac{1}{2 v}
$$

onde $\omega=\omega(\eta)=[g(\eta)]^{-1}$. Usando, $\mu=-\frac{\eta}{2 v}$ como um fator integrante da equação (94) tem-se

$$
g(\eta)=\frac{1}{1+C \exp \left(\frac{\eta}{2 v}\right)} .
$$

A constante de integração $C$ pode ser determinada pela condição de Hankine-Hugoniot (TORO, 2009, p. 71) dada conforme equação (21) para obter a reta de choque $x=\frac{1}{2} t$, onde $v=\frac{1}{2}$ é a velocidade de choque. Como as soluções de viscosidade da equação (13) sobre retas características são constantes e iguais às suas inclinações e como o caminho de choque tem inclinação $u_{0}=\frac{1}{2}$, a solução da equação (14) é ao longo dessa característica, igual a $u^{v}(x, t)=\frac{1}{2}$, quando $v \rightarrow 0$. Logo, $u^{v}(0,0)=g(0)=\frac{1}{2}=\frac{1}{1+C}$, isto é, $C=1$. Portanto, a solução da equação de Burgers em onda viajante é dada pelas equações (22) e (95) na forma

$$
u^{v}(x, t)=g(\eta)=g\left(x-\frac{1}{2}\right)=\frac{1}{1+\exp \left(\frac{2 x-t}{4 v}\right)} .
$$

A equação (96) satisfaz as condições iniciais dadas pela equação (93). De fato, se $2 x-t<0$ então $x<0$ para $t=0$, daí quando $v \rightarrow 0$ tem-se $u^{v}(x, 0) \rightarrow 1$. Contrariamente, se 
$2 x-t>0$ então $x>0$ para $t=0$, daí quando $v \rightarrow 0$ tem-se $u^{v}(x, 0) \rightarrow 0$. Retirando-se a reta de choque $x=\frac{1}{2} t$ tem-se a solução da equação de Burgers invíscida dada pela função descontínua de classe $C^{1}$ (por partes), por

$$
u=u_{v \rightarrow 0}^{v}(x, t)= \begin{cases}1 & x<\frac{t}{2}, \\ 0 & x<\frac{t}{2} .\end{cases}
$$

Esse exemplo foi abordado por Biezuner (2010, p. 75) de forma sucinta com um erro na equação (94), possivelmente devido a digitação, bem como, a omissão da obtenção da constante $C$ na equação (95). Das equações (31) e (93) tem-se

$$
\theta_{0}(x)= \begin{cases}C_{0} \exp \left(-\frac{x}{2 v}\right) & x<0, \\ C_{0} & x>0\end{cases}
$$

Da equação (34) tem-se

$$
\theta(x, t)=\frac{1}{2(\pi v t)^{\frac{1}{2}}} \int_{-\infty}^{\infty} \exp \left[-\frac{(x-\xi)^{2}}{4 v t}\right] \theta_{0}(\xi) d \xi
$$

então,

$$
\theta(x, t)=\frac{C_{0}}{2(\pi v t)^{\frac{1}{2}}} \int_{-\infty}^{0} \exp \left[-\frac{(x-\xi)^{2}}{4 v t}\right] \exp \left(-\frac{\xi}{2 v}\right) d \xi+\int_{0}^{\infty} \exp \left[-\frac{(x-\xi)^{2}}{4 v t}\right] d \xi
$$

Essas integrais já foram analisadas em exemplos anteriores, daí e usando a equação (4) resulta

$$
u(x, t)=\frac{\exp \left(\frac{-2 x+t}{4 v}\right)\left[1+\operatorname{erf}\left(\frac{-x+t}{2 \sqrt{v t}}\right)\right]}{\exp \left(\frac{-2 x+t}{4 v}\right)\left[1+\operatorname{erf}\left(\frac{-x+t}{2 \sqrt{v t}}\right)\right]+1+\operatorname{erf}\left(\frac{x}{2 \sqrt{v t}}\right)} .
$$

Quando $t \rightarrow 0$ as condições iniciais dadas pela equação (93) são válidas. Para ver isso basta usar as propriedades $\operatorname{erf}( \pm \infty)= \pm 1$. A Fig. 6 mostra o perfil das soluções da equação de Burgers obtida pela equação (96) denotada por $u^{v}$, e pela equação(101) denotada por $u$, ambas no instante $t=0,02$. 

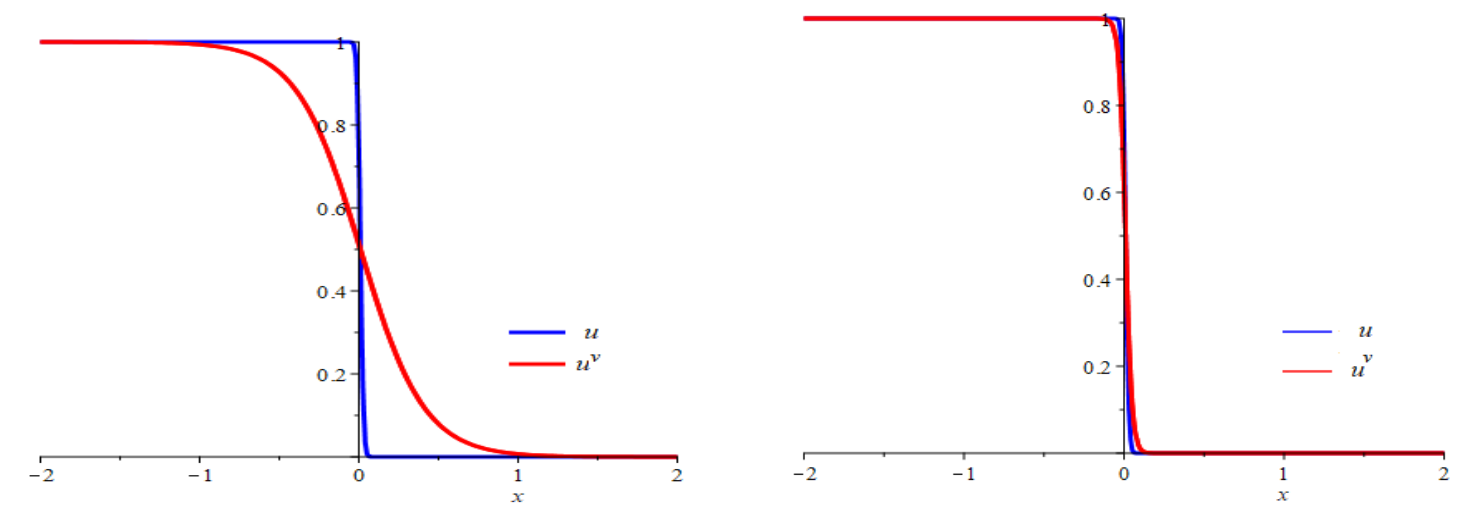

Figura 7: Curvas soluções da equação de Burgers para: (a) $R=10\left(u^{v}\right), R=100(u)$ e (b) $R=100\left(u^{v} \mathrm{e} u\right)$.

É possível notar que à medida que $v \rightarrow 0$, ou $R \rightarrow \infty$, as soluções da equação de Burgers são iguais, ou dito de outro modo, a solução $u(x, t)$ obtida como na equação (101) é no limite quando $v \rightarrow 0$ uma "onda viajante".

\section{Conclusões}

Foram analisados, a partir da solução da equação de Burgers unidimensional em um meio infinito com condição inicial por partes, três modelos teóricos não referenciados na literatura, com decaimento inicial de turbulência em um meio infinito e semi-infinito, bem como, um exemplo em meio finito de uma perturbação inicial cujo efeito mostra uma predominância do termo difusivo da equação. A solução de um exemplo de equação de Burgers com apenas um salto de descontinuidade na forma de uma onda viajante pode ser comparada com a solução obtida da mesma equação, usando a transformação de Hopf-Cole.

\section{Referências}

ÁVILA, G. Cálculo: das funções de múltiplas variáveis. 7 ed. Rio de Janeiro: LTC, 2006. v. 3.

BENTON, E. R.; PLATZMAN, G. W. A table of solutions of the one-dimensional Burgers equation. Quarterly of applied mathematics, v. 30, n. 2, p. 195-212, 1972.

BIEZUNER, R. J. Equações diferenciais parciais I/II. [Belo Horizonte]: UFMG, 2010. Notas de Aula. Disponível em: http://arquivoescolar.org/bitstream/arquivo-e/151/1/edp.pdf. Acesso em: 10 dez. 2018.

BOYCE, W. E.; DIPRIMA, R. C. Equações diferenciais elementares e problemas de valores de contorno. 6. ed. Rio de Janeiro: LTC, 1999.

BURGERS, J. M. A mathematical model illustrating the theory of turbulence. Advances in Applied Mechanics, v. 1, p. 171-199, 1948. 
CALDWELL J.; SMITH, P. Solution of Burgers' equation with a large Reynolds number. Applied Mathematical Modelling, v. 6, n. 5, p. 381-385, 1982.

CARSLAW, H. S.; JAEGER, J. C. Conduction of heat in solids. 2. ed. Oxford: Clarendon Press, 1959.

COLE, J. D. On a quasi-linear parabolic equation occurring in aerodynamics. Quarterly of Applied Mathematics, v. 9, n. 3, p. 225-236, 1951.

CUMINATO, J. A.; MENEGUETTE JUNIOR, M. Discretização de equações diferenciais parciais: técnicas de diferenças finitas. Rio de Janeiro: SBM, 2013.

DHAWAN, S.; KAPOOR, S.; KUMAR, S.; RAWAT, S. Contemporary review of techniques for the solution of nonlinear Burgers equation. Journal of Computational Science, v. 3, n. 5, p. 405-419, 2012.

FOX, R.W.; PRITCHARD, P. J.; MCDONALD, A. T. Introdução à mecânica dos fluidos. Tradução e revisão técnica de Ricardo Koury e Luiz Machado. 7. ed. Rio de Janeiro: LTC, 2015.

GUIDORIZZI, H. L. Um curso de cálculo. 6. ed. Rio de Janeiro: LTC, 2019. v.3.

HON, Y.C.; MAO, X. Z. An efficient numerical scheme for Burger's equation. Applied Mathematics and Computation, v. 95, n. 1, p. 37-50, 1998.

HOPF, B. The partial differential equation $u_{t}+u u_{x}=\mu_{x x}$. Communications on Pure and Applied Mathematics, v. 3, n. 3, p. 201-230, 1950.

KUTLUAY, S., BAHADIR, A. R.; ÖZDEŞ, A. Numerical solution of one-dimensional Burgers equation: explicit and exact-explicit finite difference methods. Journal of Computation and Applied Mathematics, v. 103, n. 2, p. 251-261, 1999.

LEVEQUE, R. J. Numerical methods for conservation laws. 2nd. ed. Basel: Birkhauser, 1992 (Lectures in Mathematics).

MITTAL, R.C.; SINGHAL, P. Numerical solution of Burger's equation. Communications in Numerical Methods in Engineering, v. 9, n. 5, p. 397-406, 1993.

ROMAO, E. C.; MARTINS, J. A.; MOURA, L. F. M. Solução numérica da equação de Burgers pelo método das diferenças centrais de sexta-ordem. Revista Iberoamericana de Ingenieria Mecânica, v. 17, n. 2, p. 117-124, 2013.

WILLIAMSON, R. E.; CROWELL, R. H.; TROTTER, H. F. Cálculo de funções vetoriais. Rio de Janeiro: LTC, 1972. v. 2.

TORO, E. F. Riemann solvers and numerical methods for fluid dynamics: a practical introduction. 3rd. ed. Berlin: Springer-Verlag, 2009. 